\title{
A comprehensive characterization of myocardial and vascular phenotype in pediatric chronic kidney disease using cardiovascular magnetic resonance imaging
}

Mun Hong Cheang ${ }^{1,2}$, Nathaniel J. Barber ${ }^{1,2}$, Abbas Khushnood ${ }^{1,2}$, Jakob A. Hauser ${ }^{1,2}$, Gregorz T. Kowalik', Jennifer A. Steeden ${ }^{1}$, Michael A. Quail ${ }^{1,2}$, Kjell Tullus ${ }^{2}$, Daljit Hothi ${ }^{2}$ and Vivek Muthurangu $u^{1,2^{*}}$

\begin{abstract}
Background: Children with chronic kidney disease (CKD) have increased cardiovascular mortality. Identifying high-risk children who may benefit from further therapeutic intervention is difficult as cardiovascular abnormalities are subtle. Although transthoracic echocardiography may be used to detect sub-clinical abnormalities, it has well-known problems with reproducibility that limit its ability to accurately detect these changes. Cardiovascular magnetic resonance (CMR) is the reference standard method for assessing blood flow, cardiac structure and function. Furthermore, recent innovations enable the assessment of radial and longitudinal myocardial velocity, such that detection of sub-clinical changes is now possible. Thus, CMR may be ideal for cardiovascular assessment in pediatric CKD. This study aims to comprehensively assess cardiovascular function in pediatric CKD using CMR and determine its relationship with CKD severity.

Methods: A total of 120 children (40 mild, 40 moderate, 20 severe pre-dialysis CKD subjects and 20 healthy controls) underwent CMR with non-invasive blood pressure (BP) measurements. Cardiovascular parameters measured included systemic vascular resistance (SVR), total arterial compliance (TAC), left ventricular (LV) structure, ejection fraction (EF), cardiac timings, radial and longitudinal systolic and diastolic myocardial velocities. Between group comparisons and regression modelling were used to identify abnormalities in CKD and determine the effects of renal severity on myocardial function.
\end{abstract}

Results: The elevation in mean BP in CKD was accompanied by significantly increased afterload (SVR), without evidence of arterial stiffness (TAC) or increased fluid overload. Left ventricular volumes and global function were not abnormal in CKD. However, there was evidence of LV remodelling, prolongation of isovolumic relaxation time and reduced systolic and diastolic myocardial velocities.

Conclusion: Abnormal cardiovascular function is evident in pre-dialysis pediatric CKD. Novel CMR biomarkers may be useful for the detection of subtle abnormalities in this population. Further studies are needed to determine to prognostic value of these biomarkers.

Keywords: Chronic renal failure, Pediatrics, Arterial stiffness, Systemic vascular resistance, Hypertension, Myocardial impairment

\footnotetext{
* Correspondence: v.muthurangu@ucl.ac.uk

${ }^{1}$ Centre for Cardiovascular Imaging, UCL Institute of Cardiovascular Science,

30 Guilford Street, London WC1N 1EH, UK

${ }^{2}$ Great Ormond Street Hospital, London, UK
} 


\section{Background}

Cardiovascular events are a leading cause of death in pediatric chronic kidney disease [1] (CKD). Twodimensional transthoracic echocardiography (TTE) is conventionally used to assess the heart in children with CKD. Several TTE markers have been used as indicators of increased risk including: left ventricular (LV) hypertrophy (LVH), LV dysfunction and abnormal myocardial strain [2-4]. However, TTE can be hampered by inaccurate measurement of volumes and mass [5], as well as operator dependence.

Cardiovascular magnetic resonance (CMR) cine imaging is the reference standard method of assessing LV volumes, ejection fraction (EF) and mass [6]. Novel CMR including tissue phase mapping (TPM) to assess myocardial velocities [7] and high temporal resolution phase contrast CMR (PCMR) to measure mitral inflow velocities and cardiac timing intervals are also now available. Furthermore, CMR can be used to characterize the vasculature by combining flow measurements with simultaneously acquired blood pressure (BP) [8]. Thus, CMR offers a comprehensive method of assessing myocardial and vascular phenotype in pediatric CKD.

The objectives of this study were to i) evaluate any differences in vascular and cardiac phenotype between healthy children and those with CKD, ii) determine the relationship between vascular and cardiac phenotype and any additional effect of $C K D$, iii) assess the relationship between markers of renal disease and cardiovascular phenotype.

\section{Methods}

\section{Study population}

The study population consisted of 120 children: 100 with confirmed CKD (stages 1-5) and 20 healthy volunteers. The CKD patients were divided into 3 groups based on CKD stage: Mild (CKD stage 1-2), Moderate (CKD stage 3 ) and Severe (CKD stage 4-5). The study was powered to detect differences in mean arterial BP (MBP) between the groups (including healthy controls). For between group comparisons, a sample size of 14 children per group provided $90 \%$ power $(p<0.05)$ to detect a difference of $3 \mathrm{mmHg}$ in MBP (assuming a normal MBP of $82 \mathrm{mmHg}$, and a variance of $40 \mathrm{mmHg}$ [9]). The group size was increased to 20 for redundancy. In addition, we increased the population size of the mild and moderate CKD groups to 40 children in order to improve our spread of estimated glomerular filtration rate (eGFR) data points for correlation analysis.

All patients were recruited from the CKD and hypertension clinics at Great Ormond Street Hospital, London. Healthy subjects were recruited by advertising within the hospital and a detailed history was taken from the parents to ensure there was no significant medical history. Exclusion criteria were: i) age $<7$ or $>18$ years, ii) congenital structural heart disease or primary myocardial disease, iii) primary renovascular disease, iv) active vasculitis v) cardiac arrhythmia, vi) medical devices precluding CMR, vii) history of current or previous renal replacement therapy and viii) acutely deteriorating renal function. This study was approved by UK national research ethics service (National Research Ethics Service Committee London, London Bridge, reference: 15/LO/ 0213). Informed parental consent and patient assent was obtained from all participants.

All CKD participants had blood and urine tests as part of their outpatient care including: haemoglobin ( $\mathrm{Hb})$, urea, creatinine, electrolytes, calcium, phosphate, intact parathyroid hormone level (PTH), and urine albumin to creatinine ratio measurements. The eGFR was estimated by modified Schwartz formula. Neither blood nor urine tests were performed for healthy controls.

\section{Imaging protocol}

Imaging was performed on a $1.5 \mathrm{~T}$ CMR system (Avanto; Siemens Healthineers, Erlangen, Germany) with vector cardiographic gating. All CMR images were processed using in-house plug-ins developed for open-source DICOM software OsiriX [10] (Osirix Foundation, Geneva, Switzerland). All images were analyzed by the same individual, who is a CMR trained cardiologist (MC) with 5 years of CMR experience. No gadolinium- contrast was given.

\section{Left ventricular volumes and mass}

LV volumes were assessed using short axis multi-slice free-breathing real-time radial $\mathrm{k}-\mathrm{t}$ SENSE balanced steady state free precision sequence sequence [11]. Scan parameters - FOV: $\approx 350 \mathrm{~mm}$, matrix: $128 \times 128$, voxel size: $\approx 2.7 \times 2.7 \times 8 \mathrm{~mm}$, TE/TR: $\approx 1.1 / \approx 2.2 \mathrm{msec}$, Flip angle: $40^{\circ}$, acceleration factor: 8 , temporal resolution: $\approx 36 \mathrm{msec}$. LV endocardial borders were manually traced at end-diastole and end-systole to evaluate end-diastolic volume (EDV) and end-systolic volume (ESV). Stroke volume (SV) was obtained by subtracting ESV from EDV. All ventricular volumes were indexed to body surface area (BSA). LV EF was assessed SV/LVEDV) $\times 100$.

Epicardial LV borders were manually traced in enddiastole and combined with endocardial borders to obtain LV mass (LVM). The effect of body size on LV mass was controlled by both indexing to height to the power of 2.7 (LVMht $^{2.7}$ ) [12] and dividing LVM by EDV to calculate mass volume ratio (MVR) [13].

The right atrial and left atrial areas were measured in diastole from 4-chamber view (using the same sequence) and indexed to BSA. 


\section{Cardiac timing and inflow velocities}

LV outflow tract and mitral inflow velocities were assessed with a free-breathing high temporal resolution real-time UNFOLD-SENSE spiral PCMR sequence. This sequence has previously been validated against Doppler echocardiography [14]. Scan parameters - FOV: $\approx 450 \mathrm{~mm}$, matrix: $128 \times 128$, voxel size: $\approx 3.5 \times 3.5 \times 7 \mathrm{~mm}, \mathrm{TE} / \mathrm{TR}: \approx 1.97 /$ $\approx 7.41 \mathrm{msec}$, flip angle: $20^{\circ}$, VENC: $150 \mathrm{~m} / \mathrm{s}$, acceleration factor: 10 , temporal resolution: $\approx 15 \mathrm{~ms}$. The imaging plane was positioned so that both the mitral inflow and LV outflow tract were imaged in the short axis.

The mitral valve orifice and LV outflow tract were manually segmented from the resultant inflow and outflow curves to obtain the isovolumic relaxation time (IRT), isovolumic contraction time (ICT) and ejection time (ET) as previously described [14]. Myocardial performance index (Tei) was calculated as the sum of ICT and IRT divided by ET. Peak early (E) and atrial (A) diastolic velocities were measured from the inflow curves.

\section{Myocardial velocities}

LV myocardial velocities was measured using a free breathing self-navigated golden-angle spiral TPM sequence planned in the mid LV short axis view [7, 15]. Scan parameters - FOV: $\approx 400 \mathrm{~mm}$, matrix: $192 \times 192$, voxel size: $\approx 2.1 \times 2.1 \times 8 \mathrm{~mm}$, TE/TR: $\approx 3.51 / \approx 11.7 \mathrm{msec}$, flip angle: $15^{\circ}$, respiratory navigation efficiency: $30 \%$, scan time: $\approx 7-$ 8 min, temporal resolution: $\approx 23 \mathrm{msec}$.

All TPM indices were measured from a mid LV short axis slice. Each acquisition produces a magnitude image and 3 phase images (in the $x-, y-$ and $z$ - direction). The epi- and endocardial borders were manually segmented on the magnitude image for all frames and regions of interest were transferred to the accompanying phase images. From these masked phase images, radial velocity was calculated by transforming the $\mathrm{x}$ and $\mathrm{y}$-direction velocities to an internal polar coordinate system using the LV center of mass as a reference point $[7,15]$. The longitudinal velocity was taken as the z-direction velocity. Global radial and longitudinal LV velocities were calculated by averaging velocities across the segmented LV slice for a given direction. The magnitude of the peak systolic ( $\left.\mathrm{S}^{\prime}\right)$, early diastolic (E') and late diastolic (A') velocities were measured from the velocity-time curves. E'/ $A^{\prime}$ ratio was the ratio of longitudinal E' over longitudinal $A^{\prime}$ velocity. The longitudinal E/E' ratio was obtained by dividing the mitral $\mathrm{E}$ wave velocity by longitudinal peak early diastolic velocity.

\section{Aortic flow}

Aortic flow assessment was performed using a breath held retrospectively-gated, spiral SENSE PCMR just above the sinotubular junction [16]. Scan parameters FOV: $\approx 400 \mathrm{~mm}$, matrix: $256 \times 256$, voxel size: $\approx 1.6 \times$
$1.6 \times 5 \mathrm{~mm}, \mathrm{TE} / \mathrm{TR}: \approx 2.1 / \approx 8.0 \mathrm{msec}$, flip angle: $25^{\circ}$, acceleration factor: 3 , breath hold time: 4-8 s, temporal resolution: $\approx 32.0 \mathrm{msec}$.

The aorta was segmented using a semi-automatic vessel edge detection algorithm with manual operator correction. The SV was derived from the flow curve and multiplied by heart rate to calculate cardiac output (CO). The maximum (AoMax) and minimum (AoMin) cross-sectional areas of the ascending aorta over the cardiac cycle were also recorded.

\section{Blood pressure measurement}

Brachial systolic, diastolic and mean arterial BPs (SBP, DBP, MBP) were measured using a CMR compatible oscillometric sphygmomanometer (Datex Ohmeda; General Electric Healthcare, Boston, Massachusetts, USA) during CMR image acquisition. This enabled optimum combination of BP and flow data for calculation of vascular indices. All BP measurements were acquired with an appropriate sized arm cuff after the subject had been lying in the scanner for at least $10 \mathrm{~min}$. Pulse pressure (PP) was the difference between SBP and DBP.

\section{Measures of vascular characteristics}

Total systemic vascular resistance (SVR) was calculated by dividing MBP by cardiac output (CO) [8]. Total arterial compliance (TAC) was calculated using a two-element windkessel model described previously [8]. Briefly, aortic flow curves were inputted into the model with measured SVR. The compliance was tuned so that pulse pressure generated by the model equaled measured pulse pressure. Ascending aortic strain (AoS) was calculated as (AoMax AoMin)/AoMin (expressed as a percentage). Local arterial stiffness was assessed by calculating ascending aortic compliance $(\mathrm{AoC})=\mathrm{AoS} /$ pulse pressure [17] .

\section{Statistics}

Statistical analyses were performed using Stata 13 (StataCorp, College Station, Texas, USA). Data were examined for normality using Shapiro-Wilk test and non-normally distributed data was transformed using a zero-skewness $\log$ transform prior to analysis. Descriptive statistics were expressed as mean ( \pm standard deviation) or geometric mean ( \pm geometric standard deviation) if data was $\log$ transformed. Between CKD group differences in vascular and cardiac phenotype were assessed using analysis of variance (ANOVA) (objective 1). Levene's test was used to assess for homogeneity of variances across the groups and Welch's correction was applied for non-homogeneous variance. Six groups of statistical tests comparing healthy controls to patients were performed in the following domains - baseline characteristics, blood pressure, vascular phenotype, global cardiac structure and function, cardiac timings and mitral 
velocities, and tissue phase mapping. Each group of tests was considered a single family of statistical inferences and the family-wise error rate was controlled using Bonferroni correction. The corresponding corrected critical $p$-values are listed in the respective tables. Post-hoc pairwise comparison was performed on parameters significantly different on ANOVA testing. The Bonferroni method was used to adjust for potential Type 1 errors in the pairwise comparisons. Spearman's correlation coefficient (rho) was used to determine the relationship between specific vascular and cardiac phenotypic markers (objective 2) that were significant on ANOVA testing. Multi-variable ANOVA models were also constructed to examine the independent effect of CKD group. Spearman's correlation coefficient was also used to determine the relationship between markers of renal disease severity - eGFR, hemoglobin $(\mathrm{Hb})$ and PTH and significant abnormal cardiac and vascular indices - MVR, IRT, S ', E', MBP, SVR (objective 3). Multi-variable linear regression was used to determine the independent effect of renal severity on cardiac markers by controlling for vascular phenotype. Only patients with recent blood tests were included into models involving blood indices. A $p$ value $<0.05$ was considered statistically significant, except where Bonferroni correction was applied as indicated. Indices not statistically different to healthy subjects have been described as 'normal'.

\section{Results \\ Demographics}

The CKD cohort $(n=100)$ consisted of 40 children with mild CKD (stage 1-2: eGFR $78.6 \pm 8.8 \mathrm{ml} / \mathrm{min} / 1.73 \mathrm{~m}^{2}$ ), 40 children with moderate CKD (stage 3: eGFR $48.3 \pm$ $8.4 \mathrm{ml} / \mathrm{min} / 1.73 \mathrm{~m}^{2}$ ) and 20 children with severe CKD (eGFR $19.2 \pm 6.5 \mathrm{ml} / \mathrm{min} / 1.73 \mathrm{~m}^{2}$ ). Twenty-five (25\%) children (mild CKD: 10, moderate CKD: 9; severe CKD: 6) had a prior diagnosis of hypertension and were on treatment (Table 1). The two most common causes of CKD were congenital abnormalities of the kidney and urinary tract $(n=60,60 \%)$ and renal dysplasia $(n=$ $11,11 \%)$. All other causes represented $<10 \%$ of the population. Eight CKD children had undergone a previous nephrectomy. No children had previously received or were currently receiving renal replacement therapy as per exclusion criteria. None of the healthy subjects $(n=20)$ had any significant past medical history nor were they taking any medications. There were no differences in age, BSA or sex distribution between groups (Table 1). As expected, markers of renal function (urea, creatinine, eGFR and urine albu$\mathrm{min} /$ creatinine ratio) were worse in the latter CKD stages, with the most abnormal results observed in

Table 1 Demographics and Baseline Characteristics of study population

\begin{tabular}{|c|c|c|c|c|c|}
\hline & $\begin{array}{l}\text { Healthy Controls } \\
(n=20)\end{array}$ & $\begin{array}{l}\text { Mild CKD } \\
(n=40)\end{array}$ & $\begin{array}{l}\text { Moderate CKD } \\
(n=40)\end{array}$ & $\begin{array}{l}\text { Severe CKD } \\
(n=20)\end{array}$ & $P$-value \\
\hline Age (years) ${ }^{a}$ & $12 \pm 1.3$ & $11 \pm 1.3$ & $12 \pm 1.3$ & $13 \pm 1.3$ & 0.12 \\
\hline Sex (\% male) & $40 \%$ & $38 \%$ & $23 \%$ & $45 \%$ & 0.42 \\
\hline Height (cm) & $162 \pm 18$ & $150 \pm 17$ & $152 \pm 18$ & $151 \pm 18$ & 0.075 \\
\hline Weight $(\mathrm{kg})^{\mathrm{a}}$ & $53 \pm 1.4$ & $43 \pm 1.5$ & $45 \pm 1.5$ & $44 \pm 1.4$ & 0.22 \\
\hline Body Mass Index $\left(\mathrm{kg} / \mathrm{m}^{2}\right)^{a}$ & $20 \pm 1.1$ & $19 \pm 1.2$ & $20 \pm 1.2$ & $20 \pm 1.1$ & 0.77 \\
\hline Body Surface Area $\left(\mathrm{m}^{2}\right)$ & $1.6 \pm 0.32$ & $1.4 \pm 0.33$ & $1.4 \pm 0.35$ & $1.4 \pm 0.3$ & 0.16 \\
\hline Hemoglobin (g/L) & & $132 \pm 13$ & $133 \pm 16$ & $128 \pm 11$ & 0.57 \\
\hline Urea (mmol/L) & & $5.4 \pm 1.2$ & $8.6 \pm 2.5$ & $18.0 \pm 5.2$ & $<0.001^{\dagger}$ \\
\hline Creatinine (umol/L) $)^{a}$ & & $68 \pm 1.2$ & $117 \pm 1.2$ & $305 \pm 1.4$ & $<0.001^{\dagger}$ \\
\hline eGFR (ml/min/1.73 m²) & & $79 \pm 8.8$ & $48 \pm 8.4$ & $19 \pm 6.5$ & $<0.001$ \\
\hline Calcium-Phosphate product $\left(\mathrm{mmol}^{2} / \mathrm{L}^{2}\right)$ & & $3.4 \pm 0.45$ & $3.4 \pm 0.39$ & $3.5 \pm 0.56$ & 0.40 \\
\hline Intact parathyroid hormone (pmol/L) ${ }^{a}$ & & $3.5 \pm 3.2$ & $3.7 \pm 2.4$ & $8.5 \pm 2.4$ & 0.011 \\
\hline Urine Albumin/ Creatinine Ratio $(\mathrm{mg} / \mathrm{mmol})^{a}$ & & $4.7 \pm 3.7$ & $22 \pm 6.2$ & $261 \pm 2.5$ & $<0.001$ \\
\hline Angiotensin converting enzyme inhibitor & & $28 \%$ & $40 \%$ & $15 \%$ & 0.13 \\
\hline Calcium Channel Blocker & & $5 \%$ & $13 \%$ & $15 \%$ & 0.38 \\
\hline Beta-Blocker & & $5 \%$ & $5 \%$ & $20 \%$ & 0.09 \\
\hline Aldosterone antagonist (Spironolactone) & & $3 \%$ & $0 \%$ & $0 \%$ & 0.47 \\
\hline
\end{tabular}

Bonferroni correction applied- $p<0.003$ is considered significant

Abbreviations: CKD chronic kidney disease, eGFR estimated globular filtration rate

aLogarithmic transformation was applied

${ }^{\dagger}$ ANOVA Welch (W) test was used. $10 \%$ of CKD patients were on 2 or more anti-hypertensive agents 
the severe CKD group $(p<0.03)$. The severe CKD group also had highest parathyroid hormone (PTH) $(p=0.01)$. There was no difference in $\mathrm{Hb}$ between the groups $(p=0.57)$. Outline of blood and urine tests results of study population can be seen in Additional file 1: Table S1.

\section{Vascular characteristics}

The DBP, DBP centile and MBP were significantly different between the groups $(p<0.001$, Table 2$)$ and were elevated in all CKD groups compared to controls $(p<$ $0.05)$ on pairwise comparison. However, there were no significant differences between the CKD groups. There were also no group differences in SBP and PP $(p \geq 0.13)$, although there was a trend for SBP centile $(p=0.08)$.

The SVR was also significantly different between the groups $(p=0.004$, Table 3$)$. On pairwise comparison, both moderate and severe CKD groups were elevated compared to controls $(p \leq 0.014)$. However, there were no significant differences in SVR between the CKD groups. Importantly, there were no group differences in TAC, local measures of ascending aorta stiffness (AoS and $\mathrm{AoC}$ ) or cardiac output (Table 3).

\section{Cardiac geometry and global function}

There was a significant difference in MVR (a marker of LV remodeling) between the groups ( $p=0.003$, Table 4). On pairwise comparison, only the severe CKD group was significantly different from healthy subjects and all other CKD groups $(p \leq 0.034)$.

The MVR correlated with all measures of BP (SBP: rho $=0.29, p=0.001$; MBP: rho $=0.26, p=0.004$; and DBP: rho $=0.19, p=0.036$; Fig. 1 ). When a model was created to study the combined effect of BP and CKD group on LV remodeling, both SBP (BP with highest univariate correlation) and CKD group were independent predictors of $\operatorname{MVR}(p=0.009, p=0.008$ respectively).

The SVR correlated with MVR (rho $=0.3, p<0.001$ ), and remained an independent predictor of MVR $(p<0.001)$ in a model including CKD group $(p=0.01)$. There was no correlation between MVR and TAC $(p=0.15)$. Although LVMht $^{2.7}$ did appear higher in CKD, this did not reach statistical significance ( $p=0.12$, Table 4$)$.

There were no differences in EDV, ESV, SV, EF, RAA or LAA between the groups (Table 4).

\section{Inflow velocities and cardiac timing intervals}

The IRT (a marker of active myocardial relaxation [18]) was different across groups $(p<0.001$, Table 5$)$. On pairwise comparison, IRT was significantly elevated in moderate and severe CKD patients compared to controls $(p \leq 0.01)$. In addition, IRT was higher in severe CKD than in mild CKD patients ( $p=0.023)$.

There was a significant association between IRT and MVR (rho $=0.25, p=0.008$ ) as seen in Fig. 1. However, both MVR and CKD group were independent predictors of IRT in a multi-variable model ( $p=0.01$ and 0.007 respectively).

There were no differences in ICT, ET or Tei index between the groups. Peak transmitral $\mathrm{E}$ and $\mathrm{A}$ wave velocities and peak E/A ratio were also normal in CKD (Table 5).

\section{Tissue phase mapping}

Radial S' velocity, a marker of systolic function, was significantly different between groups $(p=0.003$, Table 6). Specifically, radial $S^{\prime}$ was lower in moderate and severe CKD compared to controls $(p \leq 0.035)$. There was no significant difference in longitudinal $S^{\prime}$ between groups.

Radial S' negatively correlated with MBP $($ rho $=-0.22$, $p=0.016$ ), DBP (rho $=-0.37, p<0.0001-$ Fig. 2 ) and SVR (rho $=-0.22, p=0.014)$, but not SBP $(p=0.52)$. Longitudinal $S^{\prime}$ did not significantly correlate with any blood pressures. A model created to study the effects of CKD and DBP (BP with highest univariate correlation) on radial systolic velocity demonstrated that DBP was an independent predictor of radial $S^{\prime}(p=0.001)$ but CKD group was not significant $(p=0.16)$. Radial $S^{\prime}$ also significantly correlated with LVEF (rho $=0.24, p=0.009$ -

Table 2 Blood pressure profile of study population

\begin{tabular}{|c|c|c|c|c|c|}
\hline & $\begin{array}{l}\text { Healthy Controls } \\
(n=20)\end{array}$ & $\begin{array}{l}\text { Mild CKD } \\
(n=40)\end{array}$ & $\begin{array}{l}\text { Moderate CKD } \\
(n=40)\end{array}$ & $\begin{array}{l}\text { Severe CKD } \\
(n=20)\end{array}$ & $P$-value \\
\hline Systolic BP (mmhg) & $109 \pm 1.1$ & $113 \pm 1.1$ & $116 \pm 1.1$ & $116 \pm 1.1$ & 0.14 \\
\hline Systolic BP percentile ${ }^{a}$ & $33 \pm 2.5$ & $58 \pm 1.7$ & $61 \pm 1.8$ & $64 \pm 1.7$ & $0.08^{\dagger}$ \\
\hline Diastolic BP (mmhg) & $53 \pm 1.2$ & $63 \pm 1.2$ & $65 \pm 1.2$ & $69 \pm 1.2$ & $<0.001$ \\
\hline Diastolic BP percentile ${ }^{a}$ & $17 \pm 2.3$ & $45 \pm 1.9$ & $48 \pm 1.8$ & $60 \pm 1.6$ & $<0.001^{\dagger}$ \\
\hline Mean BP (mmhg) & $78 \pm 7.8$ & $84 \pm 8.8$ & $87 \pm 9.9$ & $89 \pm 8.8$ & $<0.001$ \\
\hline Pulse Pressure (mmhg) & $55 \pm 13$ & $50 \pm 10$ & $51 \pm 9.5$ & $48 \pm 12$ & 0.13 \\
\hline Heart Rate $(B P M)^{a}$ & $72 \pm 1.2$ & $75 \pm 1.2$ & $72 \pm 1.2$ & $74 \pm 1.2$ & 0.75 \\
\hline
\end{tabular}

Bonferroni correction applied- $p<0.007$ is considered significant

Abbreviations: $C K D$ chronic kidney disease, $B P$ blood pressure, $B P M$ beats per minute

aLogarithmic transformation was applied

${ }^{\dagger}$ ANOVA Welch (W) test was used 
Table 3 Vascular phenotype in CKD

\begin{tabular}{|c|c|c|c|c|c|}
\hline & $\begin{array}{l}\text { Healthy Controls } \\
(n=20)\end{array}$ & $\begin{array}{l}\text { Mild CKD } \\
(n=40)\end{array}$ & $\begin{array}{l}\text { Moderate CKD } \\
(n=40)\end{array}$ & $\begin{array}{l}\text { Severe CKD } \\
(n=20)\end{array}$ & $P$-value \\
\hline$\overline{S V R}\left(\mathrm{WU} \cdot \mathrm{m}^{2}\right)^{\mathrm{a}}$ & $21 \pm 1.3$ & $24 \pm 1.2$ & $26 \pm 1.2$ & $26 \pm 1.2$ & 0.004 \\
\hline TAC $\left(\mathrm{ml} / \mathrm{mmHg} \cdot \mathrm{m}^{2} \cdot 10^{2}\right)^{a}$ & $52 \pm 1.3$ & $55 \pm 1.2$ & $54 \pm 1.3$ & $58 \pm 1.3$ & 0.56 \\
\hline AoS (\%) & $46 \pm 1.5$ & $52 \pm 1.6$ & $53 \pm 1.6$ & $51 \pm 1.5$ & 0.74 \\
\hline $\mathrm{AoC}\left(\% \mathrm{mmHg}^{-1} \cdot 10^{2}\right)^{\mathrm{a}}$ & $86 \pm 1.6$ & $108 \pm 1.6$ & $105 \pm 1.7$ & $111 \pm 1.7$ & 0.33 \\
\hline $\mathrm{CO}^{\mathrm{a}}\left(\mathrm{I} / \mathrm{min} / \mathrm{m}^{2}\right)$ & $3.6 \pm 1.2$ & $3.5 \pm 1.2$ & $3.4 \pm 1.2$ & $3.4 \pm 1.2$ & 0.61 \\
\hline
\end{tabular}

Bonferroni correction applied- $p<0.01$ is considered significant

Abbreviations: CKD chronic kidney disease, SVR systemic vascular resistance, TAC total arterial compliance. AoS ascending aortic strain, AoC ascending aortic compliance, $\mathrm{CO}$ cardiac output

a Logarithmic transformation was applied

Fig. 2), but there was no association between LVEF and longitudinal S' $(p=0.24)$.

Radial E' velocity, a marker of LV relaxation and stiffness, was significantly lower in all CKD groups compared to controls $(p \leq 0.005)$ on pairwise comparison. However, there were no significant differences between the CKD groups. There were no significant group differences in longitudinal E' velocity in CKD (Table 6). Radial E' was negatively correlated with DBP (rho $=-0.21, p=0.021$ ) and SVR (rho $=-0.27, p=0.003$ ). Two separate models were created to separately study the effects of CKD and DBP or SVR on radial E'. Although CKD group and SVR were independent determinants of Radial E' $(p<0.003$ and 0.01 , respectively), DBP was not a significant predictor $(p=0.19)$.

In addition, radial and longitudinal E' correlated negatively with IRT $(\mathrm{rho}=-0.28, p<0.003 \&$ rho $=-0.24$, $p<0.01$ respectively). Unlike IRT, neither was associated with MVR or LVMht ${ }^{2.7}(p>0.25)$.

The E'/A' was different between groups $(p=0.003$, Table 6), but was only significantly reduced in moderate CKD compared to controls and mild CKD $(p \leq 0.035)$ on pairwise comparison. There were no group differences in radial and longitudinal A' velocities or E/E'.

\section{Association between renal and cardiovascular biomarkers} Worsening eGFR was associated with higher MBP (rho $=-0.26, p=0.04)$, MVR (rho $=-0.29, p=0.02$ ) and longer IRT (rho $=-0.30, p=0.02$ ). On multi-variable regression, eGFR remained a significant determinant of $\operatorname{MVR}(\beta=-0.26, p=0.035$, $)$ and IRT $(\beta=-0.28, p=$ $0.043)$ after adjustment for $\operatorname{MBP}(p \geq 0.06)$. In a model with IRT as dependant variable, eGFR trended towards significant $(\beta=-0.25, p=0.052)$ and MVR was significant $(\beta=0.27, p=0.039)$.

Reduced $\mathrm{Hb}$ was also associated with a reduced radial function $\left(\operatorname{Rad} \mathrm{S}^{\prime}\right.$ : rho $=0.35, p<0.005, \operatorname{Rad} \mathrm{E}^{\prime}$ : rho $=$ $0.35, p<0.006)$. These relationships remained significant after adjusting for the effect of afterload (MBP or SVR) in a multi-variable regression model $\left(\operatorname{Rad} \mathrm{S}^{\prime}\right.$ models: $\mathrm{Hb}$ $p=0.001$, Rad E' models: $\mathrm{Hb} p=0.001)$. There were no other significant relationships between eGFR, PTH and $\mathrm{Hb}$ with other cardiovascular parameters.

Table 4 Cardiac structure and global function in CKD

\begin{tabular}{|c|c|c|c|c|c|}
\hline & $\begin{array}{l}\text { Healthy Controls } \\
(n=20)\end{array}$ & $\begin{array}{l}\text { Mild CKD } \\
(n=40)\end{array}$ & $\begin{array}{l}\text { Moderate CKD } \\
(n=40)\end{array}$ & $\begin{array}{l}\text { Severe CKD } \\
(n=20)\end{array}$ & $P$-value \\
\hline LVEDV $\left(\mathrm{ml} / \mathrm{m}^{2}\right)$ & $74 \pm 11$ & $68 \pm 9.1$ & $68 \pm 10$ & $65 \pm 9.7$ & 0.03 \\
\hline $\operatorname{LVESV}\left(\mathrm{ml} / \mathrm{m}^{2}\right)^{\mathrm{a}}$ & $23 \pm 1.3$ & $21 \pm 1.3$ & $20 \pm 1.3$ & $19 \pm 1.3$ & 0.13 \\
\hline $\operatorname{LVSV}\left(\mathrm{ml} / \mathrm{m}^{2}\right)^{a}$ & $50 \pm 1.1$ & $46 \pm 1.1$ & $47 \pm 1.2$ & $46 \pm 1.2$ & 0.18 \\
\hline EF (\%) & $68 \pm 4.6$ & $68 \pm 6.1$ & $69 \pm 6.7$ & $70 \pm 5.8$ & 0.72 \\
\hline LVMht $^{2.7}\left(\mathrm{~g} / \mathrm{m}^{2.7}\right)^{\mathrm{a}}$ & $21 \pm 1.2$ & $21 \pm 1.3$ & $22 \pm 1.2$ & $25 \pm 1.2$ & 0.12 \\
\hline MVR (g/ml) & $0.7 \pm 0.1$ & $0.72 \pm 0.16$ & $0.75 \pm 0.16$ & $0.87 \pm 0.17$ & 0.003 \\
\hline RA Area $\left(\mathrm{cm}^{2} / \mathrm{m}^{2}\right)$ & $12 \pm 1.8$ & $12 \pm 1.9$ & $11 \pm 2.5$ & $11 \pm 2$ & 0.69 \\
\hline LA Area $\left(\mathrm{cm}^{2} / \mathrm{m}^{2}\right)^{a}$ & $12 \pm 1.3$ & $12 \pm 1.2$ & $11 \pm 1.2$ & $11 \pm 1.2$ & 0.38 \\
\hline
\end{tabular}

Bonferroni correction applied- $p<0.006$ is considered significant

Abbreviations: CKD chronic kidney disease, LVEDV left ventricular end-diastolic volume, LVESV left ventricular end-systolic volume, LVSV left ventricular stroke volume, EF ejection fraction, LVMht(2.7) left ventricular mass indexed to height to the power of 2.7, MVR left ventricular mass to volume ratio

aLogarithmic transformation was applied 


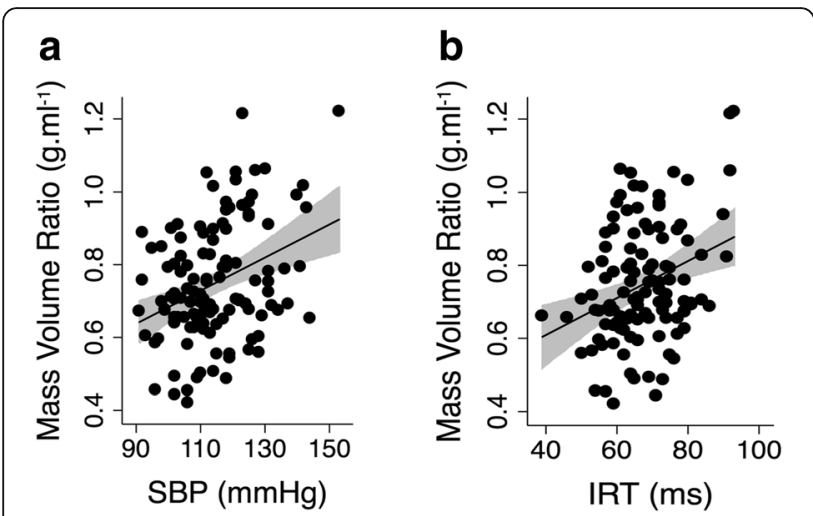

Fig. 1 a \& b: Relationship between mass volume ratio (MVR) and cardiovascular characteristics - a. MVR versus systolic blood pressure $(\mathrm{SBP})(\mathrm{rho}=0.29, p=0.001), \mathbf{b}$. MVR versus isovolumic relaxation time (IRT) (rho $=0.25, p=0.008$ ). The $95 \%$ confidence interval of the predicted mean is illustrated by grey zone

\section{Discussion}

This is the largest study to investigate the vascular and cardiac phenotype in pre-dialysis pediatric CKD using CMR. The main findings were that children with CKD had: i) elevated BP (both MBP and DBP), ii) increased SVR, but no evidence of increased vascular stiffness, iii) higher LV MVR independent of $\mathrm{BP}$, iv) abnormalities of diastolic function suggestive of reduced active relaxation and increased chamber stiffness and v) Reduced systolic velocities with preserved global systolic function. These changes appeared most marked in children with moderate and severe CKD stages.

\section{Vascular phenotype in pediatric CKD}

It is well recognized that hypertension is common in pediatric CKD [19]. However, the abnormal components of afterload have not been fully described. In this study, we found that SVR was significantly increased in all CKD groups compared to normal controls. Possible reasons include; salt retention and abnormal reninangiotensin stimulation [20], sympathetic overdrive [21] and endothelial dysfunction via reduced nitric oxide bioavailability [22]. Irrespective of the cause, our results suggest that vascular resistance is an important mediator of increased afterload and may be a useful target for intervention. Interestingly, we found no evidence of reduced TAC or increased aortic stiffness. This is slightly surprising as studies have shown elevated pulse wave velocity (PWV), reduced carotid distensibility and increased carotid intimal medial thickness in children with CKD [23]. However, elevated PWV is not a universal finding and even in positive studies, PWV is only mildly raised (i.e. in the 4C's study the PWV z-score was 0.33) [23]. Furthermore, changes in carotid artery characteristics do not necessarily reflect changes in the aorta [24], which is the repository for most vascular buffering. In fact, pulse pressure has been shown to be normal in other large pediatric CKD studies [25], which is in keeping with our finding of normal aortic stiffness. Of course, the vascular phenotype may be different in dialysis patients due to more extensive arterial calcification.

Other possible causes of hypertension in CKD are fluid overload or high CO. Although fluid overload is difficult to assess, we did show that RAA (a marker of fluid overload) was normal in our cohort. Furthermore, we also demonstrated that $\mathrm{CO}$ was not increased in CKD. Therefore, it is unlikely that these factors are important in the pathogenesis of hypertension in early CKD.

\section{Left ventricular remodelling in CKD}

In our study, we demonstrated that children in the severest CKD group had increased LV MVR compared to controls and, as eGFR fell, MVR increased. These results suggest that worsening $\mathrm{CKD}$ is associated with increased concentric remodelling in children. Interestingly, height indexed LV mass was not statistically different in our

Table 5 Cardiac timings and mitral inflow velocities in CKD

\begin{tabular}{|c|c|c|c|c|c|}
\hline & $\begin{array}{l}\text { Healthy Controls } \\
(n=20)\end{array}$ & $\begin{array}{l}\text { Mild CKD } \\
(n=40)\end{array}$ & $\begin{array}{l}\text { Moderate CKD } \\
(n=40)\end{array}$ & $\begin{array}{l}\text { Severe CKD } \\
(n=20)\end{array}$ & $P$-value \\
\hline IRT (ms) & $61 \pm 1.2$ & $64 \pm 1.1$ & $70 \pm 1.1$ & $72 \pm 1.1$ & $<0.001$ \\
\hline $\mathrm{ICT}(\mathrm{ms})^{\mathrm{a}}$ & $44 \pm 1.6$ & $36 \pm 1.6$ & $33 \pm 1.8$ & $37 \pm 1.8$ & 0.24 \\
\hline ET (ms) & $277 \pm 21$ & $275 \pm 16$ & $274 \pm 19$ & $276 \pm 15$ & 0.92 \\
\hline$T_{e}{ }^{a}$ & $0.39 \pm 1.3$ & $0.37 \pm 1.2$ & $0.38 \pm 1.2$ & $0.41 \pm 1.3$ & 0.56 \\
\hline $\mathrm{E}(\mathrm{m} / \mathrm{s})$ & $47 \pm 9.8$ & $49 \pm 11$ & $50 \pm 11$ & $49 \pm 9.7$ & 0.87 \\
\hline A (mls) & $19 \pm 4.3$ & $20 \pm 6.7$ & $21 \pm 6.8$ & $22 \pm 5.6$ & 0.36 \\
\hline E/A ratio ${ }^{a}$ & $2.6 \pm 1.2$ & $2.6 \pm 1.4$ & $2.5 \pm 1.5$ & $2.3 \pm 1.3$ & 0.63 \\
\hline
\end{tabular}

Bonferroni correction applied- $p<0.007$ is considered significant

Abbreviations: CKD chronic kidney disease, IRT isovolumic relaxation time, ICT isovolumic contraction time, ET ejection time, Tei myocardial performance index, $E$ early transmitral mean velocity, $A$ late transmitral mean velocity, $E / A$ ratio $E$ to $A$ ratio

a Logarithmic transformation was applied 
Table 6 Tissue Phase Mapping in CKD

\begin{tabular}{|c|c|c|c|c|c|}
\hline & $\begin{array}{l}\text { Healthy Controls } \\
(n=20)\end{array}$ & $\begin{array}{l}\text { Mild CKD } \\
(n=40)\end{array}$ & $\begin{array}{l}\text { Moderate CKD } \\
(n=40)\end{array}$ & $\begin{array}{l}\text { Severe CKD } \\
(n=20)\end{array}$ & $P$-value \\
\hline $\operatorname{Rad} S^{\prime}(\mathrm{cm} / \mathrm{s})$ & $2.7 \pm 0.31$ & $2.6 \pm 0.24$ & $2.5 \pm 0.38$ & $2.4 \pm 0.31$ & 0.003 \\
\hline $\operatorname{Rad} E^{\prime}(\mathrm{cm} / \mathrm{s})$ & $4.2 \pm 0.59$ & $3.6 \pm 0.62$ & $3.4 \pm 0.78$ & $3.3 \pm 0.53$ & $<0.001$ \\
\hline $\operatorname{Rad} A^{\prime}(\mathrm{cm} / \mathrm{s})$ & $1.3 \pm 0.35$ & $1.1 \pm 0.34$ & $1.2 \pm 0.31$ & $1.2 \pm 0.28$ & 0.30 \\
\hline Long S' (cm/s) & $4.6 \pm 1.6$ & $3.9 \pm 1.1$ & $3.4 \pm 1.3$ & $3.6 \pm 1.3$ & 0.009 \\
\hline Long $\mathrm{E}^{\prime}(\mathrm{cm} / \mathrm{s})$ & $7.9 \pm 1.5$ & $7.5 \pm 2.0$ & $6.7 \pm 1.8$ & $6.8 \pm 1.4$ & 0.046 \\
\hline Long A' (cm/s) & $2.3 \pm 0.71$ & $2.5 \pm 1.1$ & $2.7 \pm 0.85$ & $2.5 \pm 0.64$ & 0.61 \\
\hline Long $E^{\prime} / A^{\prime}$ ratio ${ }^{a}$ & $3.4 \pm 1.3$ & $3.2 \pm 1.6$ & $2.6 \pm 1.4$ & $2.8 \pm 1.3$ & $0.003^{\dagger}$ \\
\hline Long $\mathrm{E} / \mathrm{E}^{\prime}$ ratio ${ }^{\mathrm{a}}$ & $6 \pm 1.2$ & $6.7 \pm 1.3$ & $7.5 \pm 1.4$ & $7.3 \pm 1.4$ & 0.03 \\
\hline
\end{tabular}

Bonferroni correction applied- $p<0.006$ is considered significant

Abbreviations: CKD chronic kidney disease, Rad radial, Long longitudinal, $S^{\prime}$ systolic myocardial velocity, $E^{\prime}$ early diastolic myocardial velocity, $A^{\prime}$ late diastolic myocardial velocity, $E^{\prime} / A^{\prime} E^{\prime}$ over $A^{\prime}$ ratio, $E / E^{\prime} E$ over $E^{\prime}$ ratio

a Logarithmic transformation was applied

${ }^{\dagger}$ ANOVA Welch (W) test was used

population, unlike previous TTE studies. This may be due to the well-recognized overestimation of LV mass using TTE, particularly in patients with CKD [26].

Ventricular remodelling in these children is partly explained by their hypertensive phenotype. However, both CKD group and eGFR remained significant predictors of MVR after controlling for BP. This implies that other 'uremic' processes are involved in CKD associated remodelling. Possible 'uremic' causes of hypertrophy include: anemia, hyperparathyroidism and sympathetic overactivity $[24,27,28]$. Identification of the exact stimulus may provide new targets for therapeutic intervention that may be important in remediating the increased cardiovascular risk associated with $\mathrm{LVH}$ [2].

\section{Diastolic function in CKD}

We found conventional transmitral inflow peak E/A to be normal in children with CKD. However, both IRT and
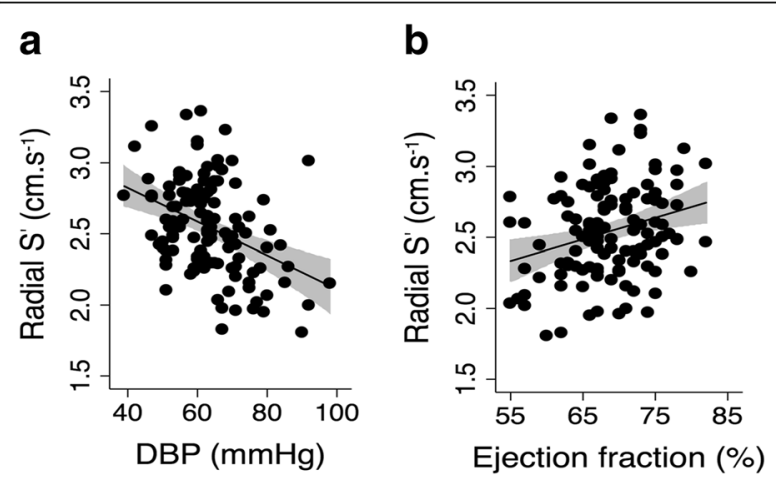

Fig. 2 a \& b: Relationship between tissue phase mapping indices and conventional measures of cardiac function - $\mathbf{a}$. Radial systolic myocardial velocity $\left(S^{\prime}\right)$ versus diastolic blood pressure (DBP) (rho $=-0.37, p<0.0001$ ), b. Radial systolic myocardial velocity (S') versus ejection function ( $r h o=0.24, p=0.009$ ). The $95 \%$ confidence interval of the predicted mean is illustrated by the grey zone radial E' were abnormal, pointing towards subtle diastolic dysfunction in moderate to severe CKD. In addition, longer IRT was associated with lower eGFR. IRT is a marker of active myocardial relaxation and correlates with invasively measured isovolumic relaxation constant [18]. Previous studies in adults have shown that active relaxation is associated with LV mass [29] and our findings are in keeping with this. However, even after controlling for MVR, CKD remains a significant predictor of IRT and eGFR trends towards significance. This suggests that CKD exerts an independent effect possibly mediated through abnormal energy or calcium handling. This could be due to myocyte/capillary mismatch resulting in a relative oxygen deficit as demonstrated in animal models [30].

Early diastolic peak velocity is a marker of both active relaxation and ventricular compliance [31]. Our demonstration of lower E, measured using TPM, is in keeping with a previous smaller study in pediatric CKD [32]. In our study, E' did not correlate with MVR or LVMht ${ }^{2.7}$. Thus, reduced early filling cannot simply be explained by LV hypertrophy. One explanation for reduced ventricular compliance is myocardial fibrosis, which has been demonstrated in several animal models [33]. Unfortunately, it is not possible to perform post contrast T1 mapping in these patients due to the risk of nephrogenic systemic fibrosis [34]. Nevertheless, non-contrast methods are now available [34] and these could be used to quantify fibrosis in future studies. We also demonstrated that lower radial E' was associated with lower $\mathrm{Hb}$. The reason for this is unclear but may be related to reduced oxygen delivery affecting the active component of relaxation.

\section{Systolic function in CKD}

Global systolic function was preserved in CKD patients with normal LVEF. However, radial $S^{\prime}$ was reduced in moderate and severe $\mathrm{CKD}$, implying some 
element of systolic dysfunction in the latter stages of renal disease. These findings are in keeping with previous tissue Doppler imaging and echocardiographic strain studies and illustrate the importance of assessing systolic function in a more sophisticated manner $[4,35]$. The most obvious explanation for reduced S' is that increased blood pressure limits contraction through the force velocity relationship. This is in keeping with our findings in which DBP independently predicted radial $S^{\prime}$, unlike CKD group.

\section{The use of CMR for cardiovascular assessment in CKD}

We have demonstrated that children with CKD exhibit a specific, but subtle cardiovascular phenotype. This phenotype is characterized by: i) increased MVR, ii) increased IRT, iv) reduced radial $S^{\prime}$ and $E^{\prime}$ and iv) increased SVR. All of these metrics can be evaluated with TTE. In fact, due to its high temporal resolution, it is probably the most accurate method of assessing timing parameters (i.e. IRT) and tissue velocity. However, there are some limitations to TTE. In particular, TTE overestimates LVM [26] and cannot accurately estimate CO (necessary for calculation of SVR). As we have found that these two measures are abnormal in pediatric CKD, we believe there may be a role for CMR identification of abnormal cardiac and vascular phenotypes in this group of patients. Nevertheless, it is still unclear what the clinical significance of this phenotype is. The next important step is to ascertain whether it can be used to guide therapy and determine prognosis.

\section{Limitations}

An important limitation is the relatively older age of the study cohort. Although the entire cohort of CKD patients did include patients from 7 to 18 years of age, the majority of children were between the ages of 9 to 16 years. Thus, the study findings may not be as applicable to younger children. In order to ensure that the study findings can be extrapolated to all age groups, future studies should consider recruiting younger patients.

The absence of recent TTE data in the subjects is also a potential limitation in this study. Performing an TTE in tandem may have been informative for the comparison of CMR findings with an established standard clinical investigation. This would have enabled us to confirm our CMR findings of subtle systolic and diastolic impairment with TTE results. Unfortunately, this was not performed as part of the study protocol in order to minimise inconvenience to the study subjects. No recent clinical TTE data were available for comparison in this cohort either. However, despite that, our findings are in agreement with the majority of previous studies in pediatric CKD using TTE as referenced in the paper.
Another limitation is our use of mild, moderate and severe CKD grouping instead of the conventional categorization of CKD stages 1-5. This was done to ensure adequate powering for group wise differences. However, the use of conventional CKD groups would be desirable in future larger, multicenter studies.

Finally, BP measurements were carried out using an oscillometric sphygmomanometer, which may be less accurate than using manual sphygmomanometer [36]. This was because it was essential for BP measurements to be acquired simultaneously during PCMR flow acquisition for accurate assessment of vascular measures. Unfortunately, this could not be performed manually in the CMR scanner during flows acquisitions. However, BP measurement was taken with a standardized protocol for all subjects using the same machine. This meant that the measurements were comparable across the cohort.

\section{Conclusion}

It is possible to use CMR to comprehensively evaluate cardiac and vascular phenotype in children with CKD. In the future, these novel CMR indices may even be useful for identifying high-risk pediatric CKD patients. More work is needed to determine normative pediatric values. Future prospective studies will also be required to correlate these markers with prognosis in order to predict cardiovascular risk.

\section{Additional file}

Additional file 1: This table outlines the clinically relevant blood and urine tests in the mild, moderate and severe CKD groups. (DOCX $17 \mathrm{~kb}$ )

\section{Abbreviations}

A: Peak late diastolic (mitral inflow) velocity; A': Peak late diastolic LV myocardial velocity; ANOVA: Analysis of variance; AoC: Ascending aortic compliance; AoMax: Maximum ascending aortic diameter; AoMin: Minimum ascending aortic diameter; AoS: Ascending aortic strain; BP: Blood pressure; BSA: Body surface area; CKD: Chronic kidney disease; CMR: Cardiovascular magnetic resonance; CO: Cardiac output; DBP: Diastolic blood pressure; E: Peak early diastolic (mitral inflow) velocity; E': Peak early diastolic LV myocardial velocity; EDV: End-diastolic volume; EF: Ejection Fraction; eGFR: Estimated glomerular filtration rate; ESV: End-systolic volume; ET: Ejection time; FOV: Field of view; Hb: Hemoglobin; ICT: Isovolumic contraction time; IRT: Isovolumic relaxation time; LV: Left ventricle/left ventricular; LVH: Left ventricular hypertrophy; LVM: Left ventricular mass; LVMht ${ }^{2.7}$ : Left ventricular mass indexed to height to the power of 2.7; MBP: Mean blood pressure; MVR: Mass volume ratio; PCMR: Phase contrast cardiovascular magnetic resonance; PP: Pulse pressure; PTH: Parathyroid hormone; PWV: Pulse wave velocity; Rho: Spearman's coefficient; S': Peak systolic LV myocardial velocity; SBP: Systolic blood pressure; SV: Stroke volume; SVR: Systemic vascular resistance; TAC: Total arterial compliance; TE: Echo time; TEl: Myocardial performance index; TPM: Tissue phase mapping; TR: Repetition time; TTE: Transthoracic echocardiography; VENC: Maximum measurable velocity range; $\beta$ : Beta coefficient of variables in regression model

\section{Acknowledgements}

We would like to express our gratitude to our research CMR radiographers and all the doctors and nurses in the renal department in Great Ormond Street Hospital, London. This work was supported by the National Institute 
for Health Research Biomedical Research Centre at Great Ormond Street Hospital for Children National Health Service Foundation Trust and University College London.

\section{Funding}

This study was funded by Kids Kidney Research, UK.

\section{Availability of data and materials}

The datasets used and analyzed during the current study are available from the corresponding author on reasonable request.

\section{Authors' contributions}

$M C$ recruited and analysed all the patient data and was a major contributor in writing the manuscript. $\mathrm{NB}, \mathrm{AB}, \mathrm{JH}, \mathrm{GK}, \mathrm{MQ}$ and JS helped with data acquisition, analysis and provided valuable support towards completion of the project. KT, DH and VM provided essential input on research hypothesis, manuscript writing and project design. All authors have read and approved the final manuscript.

\section{Ethics approval and consent to participate}

This study was approved by National Research Ethics Service Committee London, London Bridge (REC reference: 15/LO/0213). Parental and patient consent was obtained for all subjects.

\section{Consent for publication}

Permissions: All parents of participants and participants gave consent and assent to participate in the study. Permission was also obtained from parents to publish anonymized patient data collected.

\section{Competing interests}

The authors declare that they have no competing interests.

\section{Publisher's Note}

Springer Nature remains neutral with regard to jurisdictional claims in published maps and institutional affiliations.

Received: 24 May 2017 Accepted: 8 March 2018

Published online: 29 March 2018

\section{References}

1. Groothoff J, Gruppen M, de Groot E, Offringa M. Cardiovascular disease as a late complication of end-stage renal disease in children. Perit Dial Int. 2005; 25(Suppl 3):S123-6.

2. Paoletti E, de Nicola L, Gabbai FB, Chiodini P, Ravera M, Pieracci L, Marre S, Cassottana P, Luca S, Vettoretti S, et al. Associations of left ventricular hypertrophy and geometry with adverse outcomes in patients with CKD and hypertension. Clin J Am Soc Nephrol. 2016;11:271-9.

3. Hickson LJ, Negrotto SM, Onuigbo M, Scott CG, Rule AD, Norby SM, Albright RC, Casey ET, Dillon JJ, Pellikka PA, et al. Echocardiography criteria for structural heart disease in patients with end-stage renal disease initiating hemodialysis. J Am Coll Cardiol. 2016;67:1173-82.

4. Chinali M, Matteucci MC, Franceschini A, Doyon A, Pongiglione G, Rinelli G, Schaefer F. Advanced parameters of cardiac mechanics in children with CKD: the 4C study. Clin J Am Soc Nephrol. 2015;10:1357-63.

5. Hoffmann R, Barletta G, von Bardeleben S, Vanoverschelde JL, Kasprzak J, Greis C, Becher H. Analysis of left ventricular volumes and function: a multicenter comparison of cardiac magnetic resonance imaging, cine ventriculography, and unenhanced and contrast-enhanced twodimensional and three-dimensional echocardiography. J Am Soc Echocardiogr. 2014;27:292-301.

6. Pennell DJ, Sechtem UP, Higgins CB, Manning WJ, Pohost GM, Rademakers FE, van Rossum AC, Shaw LJ, Yucel EK, European society of c, Soceity for cardiovascular magnetic R. Clinical indications for cardiovascular magnetic resonance (CMR): consensus panel report. J Cardiovasc Magn Reson. 2004;6:727-65.

7. Steeden JA, Knight DS, Bali S, Atkinson D, Taylor AM, Muthurangu V. Selfnavigated tissue phase mapping using a golden-angle spiral acquisitionproof of concept in patients with pulmonary hypertension. Magn Reson Med. 2014;71:145-55.

8. Steeden JA, Atkinson D, Taylor AM, Muthurangu V. Assessing vascular response to exercise using a combination of real-time spiral phase contrast
MR and noninvasive blood pressure measurements. J Magn Reson Imaging. 2010;31:997-1003.

9. Elmenhorst J, Hulpke-Wette M, Barta C, Dalla Pozza R, Springer S, Oberhoffer R. Percentiles for central blood pressure and pulse wave velocity in children and adolescents recorded with an oscillometric device. Atherosclerosis. 2015;238:9-16.

10. Odille F, Steeden JA, Muthurangu V, Atkinson D. Automatic segmentation propagation of the aorta in real-time phase contrast MRI using nonrigid registration. J Magn Reson Imaging. 2011;33:232-8.

11. Muthurangu V, Lurz P, Critchely JD, Deanfield JE, Taylor AM, Hansen MS. Real-time assessment of right and left ventricular volumes and function in patients with congenital heart disease by using high spatiotemporal resolution radial k-t SENSE. Radiology. 2008;248:782-91.

12. de Simone G, Devereux RB, Daniels SR, Koren MJ, Meyer RA, Laragh JH. Effect of growth on variability of left ventricular mass: assessment of allometric signals in adults and children and their capacity to predict cardiovascular risk. J Am Coll Cardiol. 1995:25:1056-62.

13. Foley RN, Parfrey PS, Harnett JD, Kent GM, Murray DC, Barre PE. The prognostic importance of left ventricular geometry in uremic cardiomyopathy. J Am Soc Nephrol. 1995;5:2024-31.

14. Kowalik GT, Knight DS, Steeden JA, Tann O, Odille F, Atkinson D, Taylor A Muthurangu $V$. Assessment of cardiac time intervals using high temporal resolution real-time spiral phase contrast with UNFOLDed-SENSE. Magn Reson Med. 2015;73:749-56.

15. Knight DS, Steeden JA, Moledina S, Jones A, Coghlan JG, Muthurangu V. Left ventricular diastolic dysfunction in pulmonary hypertension predicts functional capacity and clinical worsening: a tissue phase mapping study. J Cardiovasc Magn Reson. 2015;17:116.

16. Steeden JA, Atkinson D, Hansen MS, Taylor AM, Muthurangu V. Rapid flow assessment of congenital heart disease with high-spatiotemporalresolution gated spiral phase-contrast MR imaging. Radiology. 2011;260:79-87.

17. Laurent S, Cockcroft J, Van Bortel L, Boutouyrie P, Giannattasio C, Hayoz D, Pannier B, Vlachopoulos C, Wilkinson I, Struijker-Boudier H, European network for non-invasive investigation of large $A$. Expert consensus document on arterial stiffness: methodological issues and clinical applications. Eur Heart J. 2006;27:2588-605.

18. Nagueh SF, Appleton CP, Gillebert TC, Marino PN, Oh JK, Smiseth OA, Waggoner AD, Flachskampf FA, Pellikka PA, Evangelisa A. Recommendations for the evaluation of left ventricular diastolic function by echocardiography. Eur J Echocardiogr. 2009;10:165-93.

19. Wong $\mathrm{H}$, Mylrea K, Feber J, Drukker A, Filler G. Prevalence of complications in children with chronic kidney disease according to KDOQI. Kidney Int. 2006;70:585-90

20. van Biesen W, Verbeke F, Devolder I, Vanholder R. The relation between salt, volume, and hypertension: clinical evidence for forgotten but still valid basic physiology. Perit Dial Int. 2008;28:596-600.

21. Grassi G, Quarti-Trevano F, Seravalle G, Arenare F, Volpe M, Furiani S, Dell'Oro R, Mancia G. Early sympathetic activation in the initial clinical stages of chronic renal failure. Hypertension. 2011;57:846-51.

22. Vaziri ND. Effect of chronic renal failure on nitric oxide metabolism. Am J Kidney Dis. 2001;38:S74-9.

23. Schaefer F, Doyon A, Azukaitis K, Bayazit A, Canpolat N, Duzova A, Niemirska A, Sozeri B, Thurn D, Anarat A, et al. Cardiovascular phenotypes in children with CKD: the 4C study. Clin J Am Soc Nephrol. 2017;12(1):19-28. doi: https://doi.org/10.2215/CJN.01090216.

24. Mitsnefes MM, Kimball TR, Kartal J, Witt SA, Glascock BJ, Khoury PR, Daniels SR. Cardiac and vascular adaptation in pediatric patients with chronic kidney disease: role of calcium-phosphorus metabolism. J Am Soc Nephrol. 2005:16:2796-803.

25. Sinha MD, Keehn L, Milne L, Sofocleous P, Chowienczyk PJ. Decreased arterial elasticity in children with nondialysis chronic kidney disease is related to blood pressure and not to glomerular filtration rate. Hypertension. 2015;66:809-15.

26. Arnold R, Schwendinger D, Jung S, Pohl M, Jung B, Geiger J, Gimpel C. Left ventricular mass and systolic function in children with chronic kidney disease-comparing echocardiography with cardiac magnetic resonance imaging. Pediatr Nephrol. 2016:31:255-65.

27. Mitsnefes MM, Daniels SR, Schwartz SM, Meyer RA, Khoury P, Strife CF. Severe left ventricular hypertrophy in pediatric dialysis: prevalence and predictors. Pediatr Nephrol. 2000;14:898-902. 
28. Floras JS. Clinical aspects of sympathetic activation and parasympathetic withdrawal in heart failure. J Am Coll Cardiol. 1993;22:72A-84A.

29. Muller-Brunotte R, Kahan T, Malmqvist K, Edner M, Swedish ibesartan left ventricular hypertrophy investigation vs a. Blood pressure and left ventricular geometric pattern determine diastolic function in hypertensive myocardial hypertrophy. J Hum Hypertens. 2003;17:841-9.

30. Amann K, Breitbach M, Ritz E, Mall G. Myocyte/capillary mismatch in the heart of uremic patients. J Am Soc Nephrol. 1998;9:1018-22.

31. Opdahl A, Remme EW, Helle-Valle T, Lyseggen E, Vartdal T, Pettersen E, Edvardsen T, Smiseth OA. Determinants of left ventricular early-diastolic lengthening velocity: independent contributions from left ventricular relaxation, restoring forces, and lengthening load. Circulation. 2009;119:2578-86.

32. Gimpel C, Jung BA, Jung S, Brado J, Schwendinger D, Burkhardt B, Pohl M, Odening KE, Geiger J, Arnold R. Magnetic resonance tissue phase mapping demonstrates altered left ventricular diastolic function in children with chronic kidney disease. Pediatr Radiol. 2017;47:169-77.

33. Khan R, Sheppard R. Fibrosis in heart disease: understanding the role of transforming growth factor-beta in cardiomyopathy, valvular disease and arrhythmia. Immunology. 2006;118:10-24.

34. Jellis $\mathrm{CL}$, Kwon $\mathrm{DH}$. Myocardial $\mathrm{T} 1$ mapping: modalities and clinical applications. Cardiovasc Diagn Ther. 2014;4:126-37.

35. Mencarelli F, Fabi M, Corazzi V, Doyon A, Masetti R, Bonetti S, Castiglioni L, Pession A, Montini G. Left ventricular mass and cardiac function in a population of children with chronic kidney disease. Pediatr Nephrol. 2014; 29:893-900.

36. Flynn JT, Pierce CB, Miller ER 3rd, Charleston J, Samuels JA, Kupferman J Furth SL, Warady BA, Chronic Kidney Disease in Children Study G. Reliability of resting blood pressure measurement and classification using an oscillometric device in children with chronic kidney disease. J Pediatr. 2012; 160:434-40. e431

\section{Submit your next manuscript to BioMed Central and we will help you at every step:}

- We accept pre-submission inquiries

- Our selector tool helps you to find the most relevant journal

- We provide round the clock customer support

- Convenient online submission

- Thorough peer review

- Inclusion in PubMed and all major indexing services

- Maximum visibility for your research

Submit your manuscript at www.biomedcentral.com/submit

C) Biomed Central 